\title{
Working Memory Model and Language Learning
}

\section{OPEN ACCESS}

Volume: 9

Special Issue: 2

Month: September

Year: 2021

E-ISSN: 2582-1334

Received: 10.07.2021

Accepted: 25.08.2021

Published: 15.09.2021

Citation:

Demir, Bora. "Working Memory Model and Language Learning." Shanlax International Journal of Education, vol. 9, no. S2, 2021, pp. $1-8$.

DOI: https://doi.org/10.34293/ education.v9iS2-Sep.4366

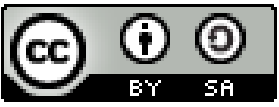

This work is licensed under a Creative Commons Attribution-ShareAlike 4.0 International License

\author{
Bora Demir \\ Çanakkale Onsekiz Mart University, Turkey \\ https://orcid.org/0000-0001-8187-3206
}

\begin{abstract}
Working Memory (WM) is an essential concept of cognitive science since many aspects of human learning depend on it. Primarily proposed by Baddeley and Hitch (1974) and developed by Baddeley (1986), the concept of WM comprises the number of subsystems involved during the process and the considerable emphasis on its key role in cognitive tasks such as learning, reasoning, and comprehension. As a complex and limited cognitive system, by enabling the input to be temporarily stored, monitored and manipulated understanding the sub mechanism under the WM model is crucial in specifying the factors that affect learning languages. By taking Baddeley's model of WM (2012) as reference, the aim of this study is to dicuss the model of WM and demonstrate the relationship between its components and the processes that play an important role in understanding the underlying cognitive processes for language learning.
\end{abstract}

Keywords: Working memory, Phonological loop, Episodic buffer.

\section{Introduction}

Learning, defined as "a process that leads to change, which occurs as a result of experience and increases the potential for improved performance and future learning" (Ambrose et al., 2010, p.3), is based on the functioning of cognitive processing of the human mind. Among many other aspects of cognitive processing involving mental procedures, memory is closely related to learning. As an essential component of learning, memory enables people to store and retrieve the information they learn.

Memory has long been classified as Short Term Memory (STM) and Long Term Memory (LTM). However, researchers in the field of educational and cognitive psychology have been discussing a relatively new term which is called Working Memory (WM). According to Dehn (2008), WM supports human cognitive processing as an interface between perception, STM, LTM, and goal-directed actions. According to Conway et al., (2007, p. 3), "WM concept reflects fundamentally a form of memory, but it is more than memory, for it is memory at work, in the service of complex cognition." Similarly, Baddeley and Logie (1999, p. 15) defined the term as “... storing and processing information while performing higher-order cognitive tasks such as comprehension, learning and reasoning."

Although both STM and WM is conceptualised as distinct components of a general memory system, some researchers have used the two terms as interchangeable or consider one a subtype of the other (McDougall, Hulme, Elllis, \& Monk, 1994). Other theorists and researchers argue that WM and STM are distinguishable constructs (Gathercole, 1998; Unsworth \& Engle, 2007; Dehn, 2008). Nevertheless, it is generally acknowledged that the two concepts are distinct. While STM passively holds information, WM has stronger relationships with academic learning and with higher-level cognitive functions. 
A growing body of research has demonstrated $\mathrm{WM}$ is a crucial factor in learning languages. Despite its limited capacity, WM is anessential memory mechanism related to processing linguistic tasks including,overall language proficiency (van den Noort, Bosch \& Hugdahl 2006), vocabulary development (Daneman \& Green,1986), grammar learning (Williams \& Lovatt 2003), reasoning (Baddeley \& Logie 1999), note taking (Kiewra \& Benton, 1988), writing (Kellog, 2016), sentence processing (Felser \& Roberts, 2007), speaking (O’Brien et al. 2006), listening comprehension (Juffs \& Harrington, 2011), reading comprehension (Kane et al., 2004; Alptekin \& Erçetin, 2009), inferential understanding (Alptekin \& Erçetin, 2010; 2011), second language aptitude (Dörnyei \& Skehan; 2003).

In studies investigating individual differences in WM capacity, Engle et al. (1999) and Engle, Tuholski et al. (1999) reported that WM is significantly related to various aspects of second language learning as; reading, decoding, reading comprehension, language comprehension, spelling, following directions, vocabulary development, note-taking, written expression, reasoning, complex learning, and grade point average (as cited in Dehn, 2008, p.93).

Today, there is sufficient evidence about the presence of astrong relationship between WM and the second language learning process. Therefore, describing the underlying mechanisms of WM is crucial for defining its role in foreign/second language learning research (Jackson, 2020). Thus, to understand the subsystems underlying the complex and dynamic structure of the WM model (Baddeley, 2000), this study is an attempt to draw implications about the WM model and second/foreign language learning.

\section{Defining Working Memory}

Memory is one of the most important concepts of cognitive science since many aspects of human life depend on it. Rather than a single unitary system, memory is an array of interacting systems, each capable of encoding or registering information, storing it, and making it available by retrieval. Without this capability for information storage, we could not perceive adequately, learn from our past, understand the present, or plan for the future (Baddeley, 1999).
The recognition of memory in learning goes back to the ancient Greeks. However, it was the mid-twentieth century when psychologists were able to identify and work on distinct dimensions and functions of memory (Dehn, 2008). As a result of the debate on the types and functioning of the concept of memory, a whole range of memory models was proposed during the 1960s. These models were composed of three types of memory namely, sensory memory, short-term memory, and long-term memory. The model which received more attention was Atkinson and Shiffrin's 1968 model (Fig.1), and it contained a detailed analysis concerning the structure and functioning of human memory. This system is considered capable of manipulating information and relating it to long-term storage. Without it, the learning of new material would be impossible. (Baddeley, 1999)

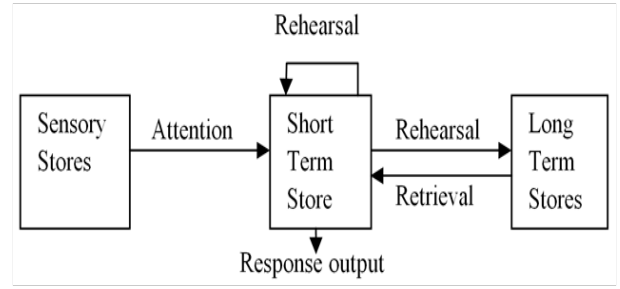

\section{Figure 1: Multi-Store Model of Memory by Atkinson and Shiffrin (1968)}

Following that, in a later study, Atkinson and Shiffrin (1971) proposed that the flow of information through the short-term storage and the subject's control of that flow of information were central to the system underlying human memory. They explained the concept of WM as a system in decision making, problem solving, and directing information flow.

Baddeley and Hitch (1974) postulated the concept of WM to overcome the problems in the early models. According to Baddeley and Hitch, this system can store and process information simultaneously. According to Baddeley and Hitch (1974), WM temporarily stores and manipulates inputto process complex tasks. Furthermore, it involves several subsystems, each related to the specific nature of the information to be processed (Baddeley, 1986). 


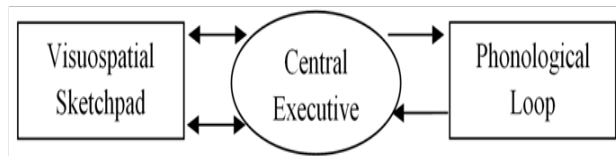

Figure 2: A schematic representation of model of WM (Baddeley and Hitch, 1974)

As mentioned above, the concept of WM was initially proposed by Baddeley and Hitch (1974) and developed by Baddeley (1986). Baddeley and Hitch proposed the model to expand a more accurate model of STM. This model was different from Atkinson and Shiffrin (1968) model since it offers a far more flexible definition of STM.

There have been conflicting views among cognitive psychologists and memory researchers on the definition and conceptualism of STM and WM. According to Baddeley (1996), the concept of WM represents a modification and extension of an earlier concept, short-term memory proposed by Atkinson and Shiffrin (1968). Baddeley differentiates the concept of WM from STM in two respects: the number of subsystems involved during the process and the considerable emphasis on its functional role in other cognitive tasks such as learning, reasoning, and comprehension.

There have been numerous efforts to conceptualise and define WM. Baddeley and Hitch (1974) argued that WM is a flexible and limited-resource system with storage and processing capabilities that exist in a traded off fashion. WM is also defined as a cognitive system that contains a limited computational space in which materials can be temporarily stored, monitored and manipulated (Baddeley, 1986; Just \& Carpenter, 1992). According to Juffs and Harrington (2011), $\mathrm{WM}$ is not an isolated term within the concept of memory.

The restricted capacity of WM limits cognitive performance (Conway et al., 2007). Individuals with larger capacity typically perform better than individuals with smaller capacity on several cognitive tasks including, complex learning, reading and listening comprehension. Oberauer et al. (2003) also define WM as a set of limiting factors for performance in complex cognitive tasks. As said by Miyake and Shah (1999), although there is the variety of definitions of WM, there is consensus among WM researchers that it comprises those mechanisms or processes that are involved in the control, regulation, and active maintenance of task-relevant information in the service of complex cognition. Hulme and Mackenzie (1992) defined the concept as the use of temporarily stored information to perform more complex cognitive tasks. In general, WM is viewed as a comprehensive system that unites various shortand long-term memory subsystems and functions (Baddeley, 1986).

One of the greatest accomplishments of the human mind is perhaps its ability to mentally maintain information in an active and readily accessible state, and selectively process new information simultaneously (Conway, Jarrold, Kane, Miyake \& Towse, 2007). In this context, WM refers to the mental processes responsible for the temporary storage and manipulation of information in the course of on-going processing (Juffs \& Harrington, 2011).

\section{The Working Memory Model and Learning}

Memory models in modern terms first started with the Atkinson-Shiffrin's Multi-Store Model of Memory (1968) as an elaboration of the information processing model proposed by Broadbent (1958) and was followed by the Levels of Processing Model of Memory (Craig \& Lockhart, 1972), and finally the Working Memory Model (Baddeley \& Hitch, 1974).

According to Baddeley (1986), WM is a system that holds and manipulates input when performing cognitive tasks such as language learning (Baddeley, 1986). According to Baddeley and Hitch (1974), during the performance of a task that involves two different systems, such as the articulatory control system and the visuospatial sketchpad, they can process both together interactively, or they can do either on its own. Baddeley and Hitch provide this argument as evidence that supports the idea that WM is composed of different components. The separation of storage from processing is the key feature that distinguishes the WM model from other models: the phonological loop, the visuospatial sketchpad and the central executive, which controls the other two subsystems, referred to as slave systems. 


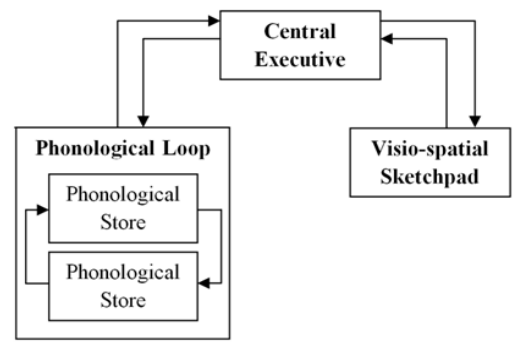

Figure 3: Conceptualization of WM model (Baddeley \& Hitch, 1974)

In 2012, Baddeley and Hitch expanded this model with a new component, multimodal episodic buffer. According to Baddeley and Hitch (1974), one of these buffers is for verbal, one for spatial information, one for visual, and one for episodic information (Baddeley, 2012). The model comprises three subcomponents, namely the visuospatial sketchpad, the episodic buffer, and the phonological loop. Figure 4 illustrates Baddeley's 2012 model of WM that contextualises the theoretical framework of the present study.

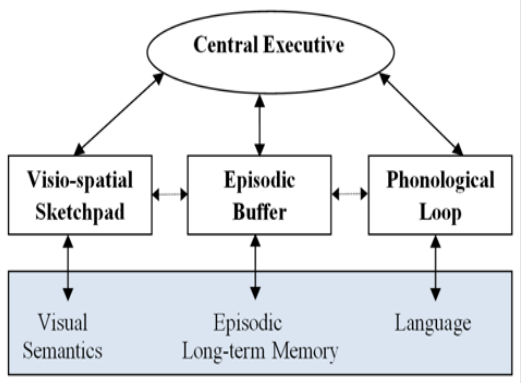

Figure 4: Baddeley's model of WM (2012)

\section{The Phonological Loop}

The PL is one of the three essential mechanisms of the WM Model. Particularly, it is responsible for the processing of verbal and acoustic input during language learning. The terminology of the term has been evolved since the earlier conception of the component was named by Baddeley and Hitch (1974) as 'phonemic buffer' and as 'articulatory loop' by Baddeley (1986). Basically, as a part of the $\mathrm{WM}$, the PL handles phonological information and rehearses verbal input. According to most models of short-term memory, one characteristic frequently assigned to short-term memory is its reliance on speech coding. Baddeley separated this aspect of memory from the rest and postulated the PL as a slave system (Baddeley, 1999).

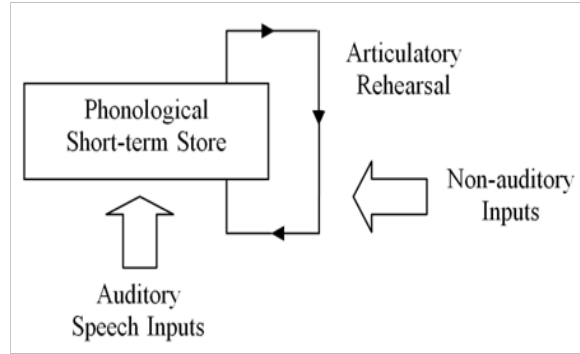

Figure 5: The Phonological Loop Model (Baddeley, 1986)

Today, it is well established that much more than a slave system, the PL may have a significant role in vocabulary acquisition. Also, it is vital for learning a second language (Miyake and Shah, 1999). Besides, the PL has a central role in language processing, literacy, and learning by facilitating the acquisition of a language (Baddeley, 2003a).

\section{The Visuospatial Sketchpad}

The Visuospatial Sketchpad (VS) can be defined as a slave system that functions for the processing of visual and spatial input (Morris \& Gruneberg, 2005). While the PL deals with speech-based information, the VS deals with visual information. Baddeley (1986) hypothesised the existence of a temporary visuospatial store that is capable of retaining and manipulating images. The VS is a cognitive construct and mental process of temporarily storing visual and spatial information for online use in operations of WM. The sketchpad also displays and manipulates visual and spatial information held in long-term memory. According to Baddeley (2002), the VS functions as an interface between visual and spatial information by connecting similar input together.

According to Baddeley (2003), the VS is a system that is involved in everyday reading tasks, where it may be involved in maintaining a representation of the page and its layout that will remain stable and facilitate tasks such as moving the eyes accurately between the lines. According to Dehn (2008), the visuospatial sketchpad plays an important role during reading by visually encoding printed letters 
and words, allowing the readers to backtrack and keep their place in the text for better comprehension.

\section{The Episodic Buffer}

Baddeley described several problems for the WM model, which stem from the need to integrate information from the subsidiary systems and the LTM by allowing active holdingand operation (Baddeley, 2012). After observing some patients with amnesia, who could not encode new information in longterm memory, Baddeley realized that these patients retrieved stories that could not be storedin the PL. As a result, Baddeley added a fourth component to the model as the third slave system, called the Episodic Buffer (EB).

According to Baddeley (2000), the EB deals both with visual and speech-based information. Baddeley (2019, p. 289) defined the EB as a connector and put it: "...visual and verbal information together with their semantic associates." As for Dehn (2008), EB increases semantic information stored and processed in WM. By underlying the importance of the EB, Dehn (2008, p. 25) stated that "The episodic buffer is important for learning because it uses multimodal codes to integrate representations from components of WM and long-term memory into unitary representations."

Baddeley (2000) assumes the executive to be a purely attentional system with a role extending beyond memory function, whereas he assumes the EB to be purely mnemonic in character. The information retrieved from the buffer is through conscious awareness allowing multiple sources of information to be processed simultaneously that is crucial during learning a language.

\section{The Central Executive}

As the main component of the WM Model, the Central Executive (CE) was first introduced by Baddeley and Hitch in 1974 as a complex system used both for the storage of information along the computational processing of that information. They defined the central executive as a workspace that is flexible but limited in capacity. Since the $\mathrm{CE}$ is used for both storing and processing, greater effort is required to process information as less capacity remains for the storage of that information.
This limited capacity can be used to regulate and coordinate the flow of information within WM besides performing processing and storage operations (Morris \& Gruneberg, 1994).

Researchers presume the term $\mathrm{CE}$ as the most complex and powerful component of the WM that controls the phonological loop and the visuospatial sketchpad and relates them to long-term memory (Morris \& Gruneberg, 1994). As the central mechanism of the model, it plays the role of attending to and switching attention from one cognitive process to another. The executive is considerably more complex than either of the two slave systems, which makes it significantly harder to investigate (Baddeley, 1996).

This system is responsible for the specific functions of learning a language such as a strategy switching, selective attention, retrieval from longterm memory and dual-task coordination, which represent higher-level cognition tasks. Hence, the multi-component structure of the model provides a basic theoretical framework for understanding how higher-level cognition is supported by the human WM system (Baddeley, 1996).

\section{Conclusion}

This study aims to describe how WM and language learning are related concepts resulting from cognitive processing. For more than forty years, cognitive psychologists and educational researchers have investigated the link between memory mechanisms and language learning. Starting from childhood, people have to store information and retrieve it when necessary owing to the memory representation in the human brain. However, as a complex process, learning a language has not been fully explained, including its cognitive mechanisms. Today, it is well established that individuals' memory capacity is a robust indicator of success or failure in language learning.

As a relatively new concept when compared with STM and LTM, WM has been proved to be associated with language learning presented by a large body of research. The contribution of WM studies in the field of language learning is precious in explaining how the human brain functions when engaging in specific language learning practices such 
as reading, listening, vocabulary learning, writing, speaking, note-taking, and language comprehension.

The WM model proposed by Baddeley (2012) is essential for cognitive psychologists and language educators in understanding the concept of WM in a deeper sense and in underlying the importance of WM capacity in explaining the reasons for success or failure in learning another language.

By the research on the sub-mechanism of WM, its components, slave systems, function in various aspects of language learning, PL, for instance, has a role in the acquisition of vocabulary. Moreover, VS is involved in reading tasks which is a part of learning a language. Furthermore, EB helps to integrate visual and verbal information from STM and LTM to allow active maintenance and manipulation together with their semantic associates. Finally, as the central mechanism of the model, the CE coordinates the PL, the VS and the EB to regulate and coordinate the flow of information to switch attention from one task to another.

However, the weakness of the WM modelis still under debate. Although commonly accepted as the predominant memory model, criticisms have been raised about the WM model in terms of the interaction between its components. According to Andrade (2001), a well-specified model of WM should help researchers to explain the memory and executive components of the phenomenon of interest and to answer the questions about the relationships between those components and the other cognitive processes involved.

However, as one of its limitations, the WM model is not perfectly specified, and it is not always clear which cognitive processes are not a function of WM. The model fails to accomplish its potential as a tool for making predictions and explaining phenomena because the components of the model and their interrelationships are underspecified (Andrade, 2001).

Another weakness of the model is its simplicity. Although simplicity seems to be the strength of the WM model, it is also a limitation since it fails in reflecting the nature of real cognition and is hard to apply to phenomena outside the domain of laboratory short-term memory tasks. In other words, the components of the model are too simple to explain the everyday phenomena and do not go deep enough to find answers to the questions of deep functioning of memory.

The third weakness of the model is related to the CE since it remains the least understood component of the model. With a framework that describes the organisation of $\mathrm{CE}$ in detail, developing experimental tasks which tap a particular aspect of executive function, or even tasks that tap the slave systems without imposing on the executive would facilitate researchers to reach more reliable findings. Besides the underspecified nature of the CE, another issue under criticism is the lack of specification of the role of the $\mathrm{CE}$ in rehearsal. The $\mathrm{CE}$ component of the WM model should be redesigned to answer the question of whether rehearsal is purely a function of the slave systems, a function of the slave systems that are initiated and monitored by the $\mathrm{CE}$, or a function solely of the CE (Richardson, 1984).

\section{References}

Alptekin, Cem, and Gulcan Erçetin. "Assessing the Relationship of Working Memory to L2 Reading: Does the Nature of Comprehension Process and Reading Span Task make a Difference?" System, vol. 37, no. 4, 2009, pp. 627-639.

Alptekin, Cem, and Gulcan Ercetin. "The role of L1 and L2 Working Memory in Literal and Inferential Comprehension in L2 Reading." Journal of Research in Reading, vol. 33, no.2, 2010, pp. 206-219.

Alptekin, Cem, and Gulcan Erçetin. "Effects of Working Memory Capacity and Content Familiarity on Literal and Inferential Comprehension in L2 Reading." TESOL Quarterly, vol. 45, 2011, pp. 235-266.

Ambrose, Susan A., et al. How Learning Works: Seven Research-Based Principles for Smart Teaching. John Wiley, 2010.

Andrade, Jackie. Working Memory in Perspective. Psychology Press, 2001.

Atkinson, R.C., and R.M. Shiffrin. "Human Memory: A Proposed System and its Control Processes." Psychology of Learning and Motivation, Academic Press, 1968, pp. 89195. 
Baddeley, A. Working Memory. Oxford University Press, 1986.

Baddeley, Alan Baddeley. "The Episodic Buffer: A New Component of Working Memory?" Trends in Cognitive Science, vol. 4, 2000, pp. 417-423.

Baddeley, A.D., and G. Hitch. "Working Memory." Recent Advances in Learning and Motivation, edited by G.A. Bower, Academic Press, 1974, pp. 47-89.

Baddeley, Alan D., and Robert H. Logie. "Working Memory: The Multiple-Component Model." Models of Working Memory: Mechanisms of Active Maintenance and Executive Control, edited by Akira Miyake, et al. Cambridge University, 1999, pp. 28-61.

Baddeley, Alan. Essentials of Human Memory. Psychology Press, 1999.

Baddeley, Alan. "Working Memory and Language: an Overview." Journal of Communication Disorders, vol. 36, no. 3, 2003, pp. 189-208.

Baddeley, Alan. "Working Memory: Theories, Models, and Controversies." Annual Review of Psychology, vol. 63, 2012.

Broadbent, Donald. Perception and Communication. Pergamon Press, 1958.

Conway, Andrew, et al. "Variation in Working Memory: An Introduction." Variation in Working Memory, edited by Andrew Conway, et al., Oxford University Press, 2008.

Cowan, Nelson. "Working Memory and Attention in Language Use." The Handbook of Psycholinguistic and Cognitive Processes: Perspectives in Communication Disorders, edited by J. Guendouzi, et al., Psychology Press, 2011, pp. 75-97.

Craik, Fergus I. M., and Robert S. Lockhart. "Levels of Processing: A Framework for Memory Research." Journal of Verbal Learning and Verbal behavior, vol. 11, no. 6, 1972, pp. 671684.

Daneman, Meredyth, and Ian Green. "Individual Differences in Comprehending and Producing Words in Context." Journal of Memory and Language, vol. 25, no. 1, 1986, pp. 1-18.

Dehn, Milton J. Working Memory and Academic Learning: Assessment and Intervention. John
Wiley, 2008.

Dornyei, Zoltan, and Peter Skehan. "Individual Differences in Second Language Learning." The Handbook of Second Language Acquisition, edited by Catherine J. Doughty, and Michael H. Long, Blackwell, 2003.

Engle, Randall W., et al. "Working Memory, ShortTerm Memory, and General Fluid Intelligence: A Latent Variable Approach." Journal of Experimental Psychology: General, vol. 128, 1999, pp. 309-331.

Felser, Claudia, and Leah Roberts. "Processing whdependencies in a Second Language: A CrossModal Priming Study." Second Language Research, vol. 23, 2007, pp. 9-36.

Gathercole, S.E. "Neuropsychology and Working Memory: A Review." Neuropsychology, vol. 8, no. 4, 1994, pp. 494-505.

Gathercole, Susan E. "The Development of Memory." Journal of Child Psychology and Psychiatry and Allied Disciplines, vol. 39, no. 1, 1998, pp. 3-27.

Gruneberg, M.M., et al. Practical Aspects of Memory. Academic Press, 1994.

Gruneberg, Michael, and Peter E. Morris. "The Major Aspects of Memory." Theoretical Aspects of Memory, Routledge, 1994.

Hulme, Charles, and Susie Mackenzie. Working Memory and Severe Learning Difficulties. Lawrence Erlbaum Associates Publishers, 1992.

Juffs, Alan, and Michael Harrington. "Aspects of Working Memory in L2 Learning." Language Teaching, vol. 44, 2011.

Just, Marcel Adam, and Patricia A. Carpenter. "A Capacity Theory of Comprehension: Individual Differences in Working Memory." Psychological Review, vol. 99, no. 1, 1992, pp. 122-149.

Kane, M.J., et al. "The Generality of WorkingMemory Capacity: A Latent Variable Approach to Verbal and Visuo-Spatial Memory Span and Reasoning." Journal of Experimental Psychology, vol. 133, no. 2, 2004, pp. 189-217.

Kellogg, R.T., et al. "The Role of Working Memory in Planning and Generating Written 
Sentences." Journal of Writing Research, vol. 7, no. 3, 2016, pp. 397-416.

Kiewra, Kenneth A., and Stephen L. Benton. "The Relationship between Information-Processing Ability and Notetaking." Contemporary Educational Psychology, vol. 13, no. 1, 1988, pp. 33-44.

McDougall, Sine, et al. "Learning to Read: The Role of Short-Term Memory and Phonological Skills." Journal of Experimental Child Psychology, vol. 58, no. 1, 1994, pp. 112-133. Miyake, Akira, and Priti Shah. "Toward Unified Theories of Working Memory." Models of Working Memory: Mechanisms of Active Maintenance and Executive Control, Cambridge University Press, 1999, pp. 442481.

O'Brien, Irena, et al. "Phonological Memory and Lexical, Narrative, and Grammatical Skills in Second Language Oral Production by Adult Learners." Applied Psycholinguistics, vol. 27, no. 3, 2006.

Oberauer, Klaus, et al. "The Multiple Faces of
Working Memory - Storage, Processing, Supervision, and Coordination." Intelligence, vol. 31, no. 2, 2003, pp. 167-193.

Richardson, John T. "Developing the Theory of Working Memory." Memory \& Cognition, vol. 12, 1984, pp. 71-83.

Shiffrin, Richard M., and Richard C. Atkinson. "The Control of Short Term Memory." Scientific American, 1971.

Unsworth, Nash, and Randall W. Engle. "On the Division of Short-Term and Working Memory: An Examination of Simple and Complex Spans and their relation to HigherOrder Abilities." Psychological Bulletin, vol. 133, 2007.

Van den Noort, Maurits, et al. "Foreign Language Proficiency and Working Memory Capacity." European Psychologist, vol. 11, 2006, pp. 289-296.

Williams, John N., and Peter Lovatt. "Phonological Memory and Rule Learning." Language Learning, vol. 53, 2003, pp. 67-121.

\section{Author Details \\ Dr. Bora Demir, Çanakkale Onsekiz Mart University, Turkey,Email ID: dbora76@yahoo.com.}

